\title{
Análisis de los prejuicios del alumnado de enfermería hacia la población migrante
}

\author{
Analysis of nursing student prejudices towards the migrant population \\ Análise dos preconceitos do alunado de enfermagem em relação à população migrante
}

Como citar este artículo:

Sánchez-Ojeda MA, Segura-Robles A, Gallardo-Vigil MA, Alemany-Arrebola I. Analysis of nursing student prejudices towards the migrant population. Rev Esc Enferm USP. 2019;53:e03532. DOI: http://dx.doi.org/10.1590/S1980-220X2018050103532

\section{Daría Angustias Sánchez-Ojeda ${ }^{1}$ \\ Adrián Segura-Robles ${ }^{2}$ \\ D Miguel Ángel Gallardo-Vigil ${ }^{3}$ \\ D Inmaculada Alemany-Arrebola ${ }^{4}$}

${ }^{1}$ Universidad de Granada, Facultad de Ciencias de la Salud de Melilla, Departamento de Enfermería, Melilla, España.

${ }^{2}$ Universidad de Granada, Facultad de Educación, Economía y Tecnología de Ceuta, Departamento de Métodos de Investigación y Diagnóstico en Educación de Ceuta, Ceuta, España.

${ }^{3}$ Universidad de Granada, Facultad de Educación y Humanidades de Melilla, Departamento de Métodos de Investigación y

Diagnóstico en Educación, Melilla, España.

${ }^{4}$ Universidad de Granada, Facultad de Educación y Humanidades de Melilla, Departamento de Psicología Evolutiva y de la Educación, Melilla, España.

\section{ABSTRACT}

Objective: To determine whether nursing students have subtle or blatant prejudices towards migrants. Method: A descriptive ex post facto study was conducted. The sample consisted of 282 students and the study variables were sex, cultural origin, age, year, and contact with migrants. The Subtle and Blatant Prejudice Scale was used. Descriptive and inferential analyses were performed. Results: There were significant differences in the culture variable, with students of Berber origin and of Muslim faith showing less prejudice than those of European origin and of Christian faith. Fourth-year students had the most blatant prejudices. According to the variable contact with migrants, it is the students who have not had contact with this group who have the greatest blatant prejudices. Conclusion: Nursing students have more subtle prejudices rather than blatant prejudices towards the migrant population.

\section{DESCRIPTORS}

Students, Nursing; Migrants; Prejudice; Attitude; Nursing Care. 


\section{INTRODUCCIÓN}

El aumento del número de migrantes que ha experimentado España en pocas décadas ha provocado un cambio sustancial en la manera de pensar de sus habitantes hacia este colectivo. Si a mediados de los años 90 la población española estaba a favor de la migración y la consideraba necesaria, en la década pasada la situaba dentro de los cuatro primeros problemas del país ${ }^{(1)}$. Por lo que esta situación puede provocar cambios en las actitudes de la población ante este fenómeno y prejuicios hacia los migrantes, por lo que debe ser objeto de estudio.

Las actitudes, en un lenguaje coloquial, se definen como una postura que expresa una forma de ver el mundo y los pensamientos que tiene una persona hacia el objeto de actitud ${ }^{(2)}$. Por otro lado, el prejuicio se define como aquellos juicios evaluativos cargados de afectividad negativa cuyo objeto de esta actitud prejuiciosa es el exogrupo, siendo esta actitud de rechazo relativamente estable y duradera en el tiempo, pudiendo conllevar comportamientos de rechazo hacia los miembros del exogrupo por el simple hecho de pertenecer a ese grupo ${ }^{(3)}$.

Por esto, la aparición de actitudes prejuiciosas se produce cuando el exogrupo empieza a ser percibido por los miembros del endogrupo como una amenaza por la competencia por los recursos disponibles o por ser una amenaza para su identidad ${ }^{(4)}$, pudiéndose expresar estas actitudes de manera abierta o no.

En las sociedades occidentales no está bien visto, e incluso es denunciable, expresar abiertamente opiniones prejuiciosas hacia un determinado colectivo, en este caso hacia los migrantes. Es por ello que se ha ido modificando las formas de expresar las actitudes prejuiciosas, pasando del prejuicio manifiesto al prejuicio sutil. El primero se caracteriza por el rechazo abierto a los miembros del exogrupo debido a la percepción de amenaza a los recursos del endogrupo y al rechazo por el contacto y relaciones cercanas o íntimas. El segundo, el prejuicio sutil, se caracteriza por la defensa de los valores tradicionales, siendo el exogrupo quien no lo respetan ${ }^{(5)}$.

Entre las razones del prejuicio étnico se encuentra la saliencia perceptiva de ciertos aspectos particulares de las minorías que se convierten en informativas de cómo son y cómo se comportan los miembros del exogrupo, que siempre llevan asociadas creencias y emociones con connotaciones negativas que pretenden legitimar las actitudes prejuiciosas ${ }^{(6)}$.

Aunque la diversidad cultural existente en las sociedades actuales es fuente de enriquecimiento social, también puede conllevar problemas e inconvenientes debido al contacto entre los autóctonos y migrantes provocados por la existencia de estereotipos culturales heredados que acarrean prejuicios y discriminación ${ }^{(7)}$. Sobre la Teoría del Contacto intergrupal e interétnico, son diversos los estudios que han analizado su influencia con la reducción del estereotipo, partiendo de la hipótesis planteada por Allport en 1954, son diferentes autores $^{(8-9)}$ los que indican que existe una relación inversa entre el contacto intergrupal y la manifestación del prejuicio y de los estereotipos interétnicos.
Así, las actitudes prejuiciosas que se observan en la sociedad no son ajenas en el ámbito de la Universidad, siendo diversos los estudios realizados con estudiantes universitarios que demuestran que poseen tanto prejuicios sutiles como manifiestos hacia la migración ${ }^{(10-12)}$.

Las migraciones representan un problema mundial que repercute en todas las áreas de actuación humana, y de manera particular, en lo relacionado con la salud, por lo que el personal de enfermería se ve altamente implicado, ya que está en contacto directo con todos los pacientes, tanto autóctonos como población migrante. Poseer actitudes prejuiciosas hacia los colectivos de migrantes puede derivar en comportamientos discriminatorios. Por esto, es necesario formar a los estudiantes del Grado en Enfermería en la competencia de adaptación transcultural, ya que las raíces culturales no pueden ser obviadas, por lo que hay que cuidar diversos aspectos que pueden abarcar desde el lenguaje $e^{(13)}$ que se utiliza con los pacientes hasta los cuidados que se practican. Por lo que se debe formar en el conocimiento y la concienciación en el trato de los pacientes, estando en la base los valores universales, tales como respetar la vida, la dignidad y los derechos de todas las personas sin distinción de nacionalidad, religión, color, edad, sexo o posición social; tal y como reflejan los principios éticos de beneficencia (hacer siempre el bien), no maleficencia (evitar hacer daño), de justicia (tratar a todas las personas por igual) y el principio de autonomía (el paciente es capaz de tomar sus propias decisiones $)^{(14)}$.

Esta formación y concienciación en valores universales y de justicia social en el personal de enfermería y cuidadores son necesarias para afrontar cualquier situación en el cuidado de las personas, sin que sus creencias y prejuicios personales influyan en su trabajo ${ }^{(15)}$.

Para trabajar las actitudes prejuiciosas hacia el exogrupo es necesario investigar en contextos pluriculturales. Por esto la investigación se ha desarrollado en la ciudad de Melilla (España), situada en el norte de África donde, que en solo $12 \mathrm{~km}^{2}$ conviven principalmente cuatro culturas: cristianos de procedencia europea y religión cristiana, hebrea, gitana y bereberes de religión musulmana (grupo cultural de origen musulmán y lengua tamazight que reside en Melilla, España), siendo los grupos más numerosos el europeo y el bereber. La ciudad de Melilla presenta unas características muy particulares para la investigación de la interculturalidad, ya que su situación geográfica, ciudad fronteriza con Marruecos, hace que miles de marroquíes cruzan a diario, de forma legal, las fronteras de la ciudad para trabajar y, en algunas ocasiones, para hacer uso del único hospital que existe en la ciudad. Además, esta ciudad es una de las rutas principales de los migrantes en situación irregular que buscan llegar a Europa a través de España.

Siendo Melilla (España) una ciudad multicultural y dada la alta presión migratoria que vive en la última década, es necesario realizarse las siguientes preguntas de investigación, ¿̇presenta el alumnado del Grado en Enfermería actitudes prejuiciosas hacia los migrantes y la población transfronteriza? Si existen actitudes prejuiciosas, ¿hay diferencias en las actitudes prejuiciosas 
hacia los migrantes en función de las variables sexo, procedencia cultural, curso o contacto con miembros de este colectivo?

Dada la situación privilegiada en materia de diversidad étnica, el objetivo principal de esta investigación ha sido conocer si existen prejuicios, Sutil y Manifiesto, en los estudiantes de Enfermería de la Facultad de Ciencias de la Salud de Melilla (España) hacia el colectivo de migrantes.

\section{MÉTODO}

\section{Diseño de estudio}

Para la realización de este trabajo se ha utilizado una metodología empírico-analítica, es un estudio Ex-Post-Facto de tipo descriptivo.

En función del objetivo de estudio que se plantea se consideran las siguientes variables: variables sociodemográficas: sexo, edad, curso, cultura y contacto con inmigrantes. Variables dependientes: los prejuicios sutiles y manifiestos del alumnado del Grado en Enfermería del campus de Melilla.

\section{Populación}

El criterio de inclusión establecido para esta investigación ha sido ser estudiantes del Grado en Enfermería de la Facultad de Ciencias de la Salud del Campus de Melilla de la Universidad de Granada (España).

\section{ReColección de LOS DATOS}

Para la selección de la muestra se ha realizado un muestreo no probabilístico de tipo intencional, siendo el total de estudiantes matriculados de 387 durante el curso académico 2016/2017, la muestra invitada a participar de 325 y la muestra productora de datos, 282, lo que supone un $72,86 \%$ de la población total. Es decir, el error muestral es de 3,04\% con un nivel de confianza del 95\%.

En relación con la edad del alumnado, la media de edad es de 21,73, que varia de 17 a 50 años, agrupándose en los siguientes rangos: 107 participantes que se encuentran en el rango de 17-20 años (37,9\%), 138 universitarios con edades comprendidas entre los 21 a 24 años (48,9\%), 27 estudiantes de $25-28$ años $(9,6 \%)$ y solo 10 son mayores de 29 años $(3,5 \%)$.

En cuanto al sexo, son 223 mujeres (casi el 80\% de la muestra), lo que refleja la feminización de la profesión de Enfermería. En función de la cultura de procedencia, 211 alumnos, es decir, el 74,8\% se identifica de procedencia europea y de religión cristiana, 61 estudiantes, es decir, el 21,6\% de procedencia bereber y de religión musulmana y el 3,6\% restante (10 estudiantes) se identifica con otras culturas que no fueron tomadas en cuenta para el análisis, debido al reducido número de sujetos.

En relación con el curso, el 30,5\% son de primer curso, el 29,4\% de segundo, el 27,3\% de tercero y 12,8\% está en cuarto curso. Por último, el alumnado que ha tenido alguna vez contacto con migrantes es el $82,6 \%$ y los que no han tenido contacto el $17,4 \%$.

\section{INSTRUMENTO}

Para la obtención de los datos se ha utilizado la Escala de Prejuicio Sutil y Manifiesto ${ }^{(5)}$ validada y traducida al español $^{(16)}$. La escala mide el Prejuicio Sutil y Manifiesto a través de 10 ítem para cada subescala, siendo el formato de respuesta tipo Likert, que puntúa de 1 a 5 , siendo 1 "en total desacuerdo"(TD), 2 "en desacuerdo" (D), 3 “indiferente" (I), 4 "de acuerdo" (DA) y 5 "en total acuerdo" (TA). Tal y como hicieron los autores se recodifican los ítems que puntúan de forma inversa: 4, 7, 13, 18, 19 y 20, de tal manera que puntúan todos iguales, es decir, a mayor puntuación más prejuicios hacia la inmigración.

Esta escala ha sido ampliamente validada ${ }^{(11,16-17)}$, aunque para este estudio se procede a comprobar los valores de validación. Se calculó la fiabilidad del cuestionario a través del alfa de Cronbach, siendo este de $\alpha=, 877$ para el total. Analizadas las subescalas, los datos indican que la fiabilidad del Prejuicio Sutil es de $\alpha=, 789$ y para la Prejuicio Manifiesto es de $\alpha=.800$. Aunque este estadístico ha sido ampliamente utilizado debemos completarlo con otro tipo de análisis para evitar sesgos propios de la prueba ${ }^{(18)}$, como la Fiabilidad Compuesta (FC) y la Varianza Media Extractada (VME). Los resultados obtenidos muestran para el Prejuicio Sutil una $\mathrm{FC}=.912$ y $\mathrm{VME}=.533$; $\mathrm{y}$, para el Prejuicio Manifiesto, una $\mathrm{FC}=.911$ y $\mathrm{VME}=.524$, obteniéndose valores que dan robustez a la fiabilidad del cuestionario ${ }^{(19)}$.

Para acceder a la muestra se pidió permiso al Decanato de la Facultad y al profesorado que imparte docencia en las asignaturas donde la asistencia es obligatoria, garantizando la asistencia del mayor número de estudiantes. Además, el instrumento fue cumplimentado de manera voluntaria.

\section{ANÁLISIS Y PROCESADO DE LOS DATOS}

Para el análisis de los datos se ha empleado el paquete estadístico Statistical Package for the Social Sciences (SPSS 25) y se han realizado distintos análisis descriptivos e inferenciales. Dada que la muestra cumple con los requisitos de normalidad, homocedasticidad e independencia, se recurre al uso de pruebas paramétricas ${ }^{(20)}$, excepto en los casos en lo que las muestras no superaron los 50 sujetos que se recurre a una comparación múltiple no paramétrica, la $\mathrm{H}$ de Kruskal-Wallis ${ }^{(21)}$.

\section{Aspectos éticos}

La investigación fue aprobada por el Comité de Investigación de la Facultad de Ciencias de la Salud del Campus de Melilla de la Universidad de Granada (España), siguiendo los principios éticos que rigen las investigaciones con seres humanos. Se explicó a los alumnos que los cuestionarios eran anónimos y se les brindó una breve explicación de la confidencialidad y el consentimiento informado.

\section{RESULTADOS}

Para dar respuestas al objetivo, en primer lugar se ha calculado la puntuación total de la Escala y de las Subescalas de Prejuicio Sutil y Prejuicio Manifiesto. En la Tabla 1 se presenta junto con sus puntuaciones medias, mínimo y 
máximos y los percentiles 50 y 75. Tal como se observa en la tabla, las mayores medias se observa en el Prejuicio Sutil. Además, el 19\% de los encuestados obtienen bajas puntuaciones en la Subescala del Prejuicio Sutil (53 de los participantes) frente al $55.9 \%$ que se encuentra en el percentil más alto, es decir, 156 estudiantes obtienen puntuaciones superiores a 34 . En cuanto a la Subescala de Prejuicio Manifiesto, el 25,4\% de estudiantes obtienen puntuaciones por debajo del percentil 25 (71 alumnos) frente al 29,7\% que son los que muestran puntuaciones superiores a 30 , lo que muestra un mayor Prejuicio Manifiesto (83 alumnos el total de la muestra).

Tabla 1 - Puntuaciones de la Escala de Prejuicios Sutil y Manifiesto - Melilla, España, 2016-2017.

\begin{tabular}{|c|c|c|c|c|}
\hline & & Sutil & Manifiesto & Total \\
\hline $\mathbf{N}$ & & 282 & 282 & 282 \\
\hline$M$ & & 30,45 & 27,59 & 58,04 \\
\hline SD & & 5,79 & 3,83 & 8,41 \\
\hline Mín & & 16 & 13 & 22 \\
\hline Máx & & 47 & 41 & 78 \\
\hline \multirow{3}{*}{ PC } & 25 & 27 & 25 & 52 \\
\hline & 50 & 31 & 28 & 58 \\
\hline & 75 & 34 & 30 & 63 \\
\hline
\end{tabular}

$\mathrm{N}$ : Número de sujetos para cada tipo de prejuicio; $\mathrm{M}$ : Media de las puntuaciones obtenidas; SD: Desviación Típica; Mín: Puntuación mínima obtenida; Máx: Puntuación máxima obtenida; PC: Percentiles establecidos para la selección de los sujetos.

A continuación se realizaron los análisis inferenciales en función de las variables sexo, cultura, edad, curso, y contacto con migrantes y la relación tanto con las Escala de Prejuicio como con las Subescalas de Prejuicios Sutil y Manifiesto. En referencia con la variable sexo no existen diferencias significativas ni en la Escala total ni en la Subescala de Prejuicio Sutil. Así, prácticamente obtienen las mismas medias los hombres y las mujeres (Mmujer= 46,39; Mhombre= 46,35). Por el contrario, sí se observan diferencias significativas en la Subescala de Prejuicio Manifiesto siendo los hombres los que obtienen los mayores puntajes $(\mathrm{Mmujer}=27,36$; Mhombre $\left.=28,67 ; \mathrm{t}=-2,354 ; \mathrm{p}<, 05 ; \mathrm{d}_{\mathrm{COHEN}}=-, 35\right)$ siendo el tamaño del efecto pequeño.

En cuanto a la variable cultura, solamente se han tenido en cuenta las culturas de procedencia europea que son de religión cristiana y la de procedencia bereber, de religión musulmana, debido a que las otras no han tenido representación ( $3 \%$ del total de participantes). Como se observa en la Tabla 2 se ha encontrado diferencias estadísticamente significativas tanto en la Escala Total como en las Subescalas de Prejuicio Sutil y Manifiesto, teniendo una puntuación mayor los alumnos de procedencia europea con religión cristiana, es decir, mayor prejuicio, e inferior puntuación los alumnos de procedencia bereber con religión musulmana, siendo en todos los casos el tamaño del efecto mediano.
Tabla 2 - Resultados de la prueba t de Student en relación con la cultura - Melilla, España, 2016-2017.

\begin{tabular}{|c|c|c|c|c|c|c|c|}
\hline Escala & & $\mathbf{N}$ & $M(D T)$ & $\mathbf{t}$ & $\boldsymbol{P}$ & $\mathbf{d}_{\text {COHEN }}$ & $\begin{array}{c}\text { Efecto del } \\
\text { tamaño }\end{array}$ \\
\hline \multirow{2}{*}{$\begin{array}{l}\text { Prejuicio } \\
\text { Total }\end{array}$} & Europea & 208 & $47,68(11,14)$ & \multirow[b]{2}{*}{3,61} & \multirow{2}{*}{, $000 * *$} & \multirow{2}{*}{, 526} & \multirow{2}{*}{252} \\
\hline & Bereber & 61 & $41,77(11,29)$ & & & & \\
\hline \multirow{2}{*}{$\begin{array}{l}\text { Prejuicio } \\
\text { Sutil }\end{array}$} & Europea & 208 & $27,94(6,47)$ & \multirow{2}{*}{3,56} & \multirow{2}{*}{, $000^{* *}$} & \multirow{2}{*}{, 527} & \multirow{2}{*}{, 255} \\
\hline & Bereber & 61 & $24,52(6,49)$ & & & & \\
\hline \multirow{2}{*}{$\begin{array}{l}\text { Prejuicio } \\
\text { Manifiesto }\end{array}$} & Europea & 208 & $22,13(5,88)$ & \multirow{2}{*}{2,96} & \multirow{2}{*}{, $003^{*}$} & \multirow{2}{*}{, 566} & \multirow{2}{*}{272} \\
\hline & Bereber & 61 & $19,42(3,34)$ & & & & \\
\hline
\end{tabular}

$\mathrm{N}$ : Número de sujetos en la comparación; M: Media para cada una de las muestras seleccionadas; DT: Desviación típica; t: Test t de Student; p: Significación bilateral $* *<.001 ; *<.005$

En referencia a la edad solo se han encontrado diferencias significativas con esta variable en la Subescala de Prejuicio Sutil, siendo el alumnado de edades comprendidas entre los 21 a 24 años los que obtienen los puntajes más altos y los más bajos los estudiantes mayores de 28 años (M2124años=31,28; M25-28años=30,62; M17-20años= 29,66; $\mathrm{M}+28 \mathrm{años}=26,77 ; \mathrm{F}=2,89 ; \mathrm{p}<, 05)$.

Seguidamente se analiza la variable curso,los datos indican que no se existen diferencias significativas ni en la Escala de Prejuicio Total ni en las subescalas de Prejuicio Sutil y Manifiesto, aunque conforme avanza el curso aumentan las puntuaciones tanto del Prejuicio Manifiesto como es en la Escala Total, siendo el alumnado del último curso el que obtiene mayores puntajes.

Para analizar la variable contacto con migrantes, en este caso, los que sí mantienen un contacto habitual con los que no lo mantienen (Tabla 3). Los datos indican que solo existen diferencias significativas en la Subescala de Prejuicio Manifiesto. Así, los estudiantes que sí han mantenido contacto con los migrantes obtienen una puntuación menor que los estudiantes que no han mantenido ningún contacto con ellos, siendo en ambos casos el tamaño del efecto pequeño.

Tabla 3 - Descriptivos y prueba t en función del contacto con migrantes - Melilla, España, 2016-2017.

\begin{tabular}{lcccccc}
\hline & Contacto (N) & $\mathbf{M}$ & SD & $\mathbf{t}$ & $\boldsymbol{P}$ & $\mathbf{d}_{\text {COHEN }}$ \\
\hline $\begin{array}{l}\text { Prejuicio } \\
\text { Sutil }\end{array}$ & Sí (230) & 27 & 6,90 & & & \\
\hline Prejuicio & No (49) & 27,93 & 5,93 &,- 884 &, 337 & -- \\
Manifiesto & Sí (230) & 21,10 & 6,57 & & & \\
\hline Prejuicio & No (49) & 23,79 & 5,64 & $-2,665$ &, $008^{*}$ &,- 43 \\
Total & Sí (230) & 45,78 & 11,91 & & & \\
\hline
\end{tabular}

N: Participantes; M: Media para cada una de las muestras seleccionadas; SD: Desviación típica; $\mathrm{t}$ : Test $\mathrm{t}$ de Student; $\mathrm{p}$ : Significación bilateral $\mathrm{p} *<.05$.

\section{DISCUSIÓN}

En primer lugar, los datos obtenidos en la Escala de Prejuicio Sutil y Manifiesto muestran que el cuestionario es válido y fiable, en la línea de otros estudios realizados ${ }^{(5,11,16-17,22)}$, 
siendo mayor en la Subescala de Prejuicio Sutil que en la de la Subescala de Prejuicio Manifiesto.

Por otro lado, los estudiantes puntúan más alto en la Escala de Prejuicio Sutil que en la Escala de Prejuicio Manifiesto, hecho que coincide con el estudio realizado por otros autores ${ }^{(10-11)}$ con estudiantes universitarios, lo que vuelve a mostrar la transformación de un prejuicio manifiesto y abierto a otro más sutil y encubierto ${ }^{(5,16,23)}$, siendo una posible explicación la deseabilidad social del alumnado que quiere mostrarse abierto y sin prejuicios.

En cuanto al género, se aprecian diferencias significativas en la Subescala Prejuicio Manifiesto siendo los hombres los que han puntuado más alto que las mujeres, resultado coincidente con otras investigaciones ${ }^{(24)}$, estos datos indican que los hombres presentan actitudes más prejuicios que las mujeres. Este resultado debe ser tenido en cuenta y trabajar desde la formación inicial en el Grado en Enfermería para reducir o minimizar las creencias negativas que puede conllevar estereotipos y prejuicios hacia este colectivo.

De las dos culturas más representativas de la muestra de estudio, europea y bereber, se ha encontrado diferencias significativas tanto en la Escala Total como en las dos Subescalas, siendo los universitarios de origen bereber menos prejuiciosos que los de origen europeo, dato coincidente con otro estudio realizado en el mismo contexto a estudiantes universitarios $^{(11)}$. Como posible explicación podría ser que los alumnos bereberes pueden identificarse con los migrantes de la ciudad, ya que comparten aspectos culturales como la religión, por lo que presentan mayor identidad social con este colectivo ${ }^{(25)}$.

En cuanto a la variable curso, no se han hallado diferencias significativas aunque la tendencia es que conforme avanza el curso académico aumentan las actitudes prejuiciosas del alumnado, siendo el estudiante del último curso de grado el que obtiene mayores medias. Este dato no coincide con otros estudios, en el que el alumnado que está realizando las prácticas hospitalarias al entrar en contacto con los migrantes los niveles de prejuicio se reducen significativamente $^{(26-27)}$. Dado los resultados encontrados en los universitarios de cuarto curso, sería conveniente diseñar e implantar programas, cursos o seminarios de educación en valores a lo largo de la carrera para desarrollar competencias culturales hacia la diversidad durante esta etapa de formación práctica, para incidir en la ética en la enfermería y deconstruir las actitudes prejuiciosas hacia este exogrupo que redunde en un trato justo al paciente independiente del sexo, religión, cultura o etnia ${ }^{(28)}$.

$\mathrm{Al}$ analizar la muestra en función de la variable contacto directo con los migrantes que tienen los estudiantes, se ha encontrado diferencias significativas en la Subescala de Prejuicio Manifiesto, siendo el alumnado que ha vivenciado el contacto los menos prejuiciosos frente a los que no han tenido contacto nunca con este colectivo. Este hecho coincide con la Teoría del Contacto Intergrupa ${ }^{(29)}$ que postula que el contacto con el exogrupo es una condición para disminuir la hostilidad intergrupal. Esta Teoría está reforzada por numerosos estudios ${ }^{(30-33)}$, aunque deberíamos profundizar en estos resultados, ya que en el cuestionario solamente se pregunta sobre la existencia de contacto con el colectivo de migrantes, pero no se especificaba qué tipo de contacto (amistad, compañeros, vecinos...).

\section{CONCLUSIÓN}

Para concluir, los datos indican que el alumnado del Grado en Enfermería presenta actitudes prejuiciosas, siendo mayor las puntuaciones obtenidas en la Subescala de Prejucio Sutil. Además, que las mujeres presentan menos prejuicios hacia la población migrante que los hombres, aunque los puntajes aumentan conforme aumenta el curso académico. Dato que deberá ser tenido en cuenta para incluir, en los planes de estudio en la formación de los futuros profesionales de la salud, materias relacionadas con la diversidad cultural y la deconstrucción de estereotipos y prejuicios que puede incidir negativamente en las creencias hacia el exogrupo.

Por otro lado, existen diferencias significativas en función de la variable cultura, siendo el alumnado de religión musulmana y procedencia bereber los que presentan menos actitudes prejuiciosas posiblemente como consecuencia de una mayor identidad cultural con los migratntes, ya que presentan similitudes en relación con la religión que profesan lo que puede redundar de forma positiva en una mayor empatía hacia el exogrupo.

Por último, existen diferencias significativas en función del contacto que han tenido el alumnado con el exogrupo, siendo positivo ya que a mayor contacto menos actitudes prejuiciosas. Por esto, sería necesario seguir profundizando en esta variable que puede ayudar a eliminar barreras mentales.

Por todo ello, los futuros profesionales de Enfermería, al igual que sucede con el resto de la población, no son impermeables a los fenómenos que suceden a su alrededor. Al tratarse de estudiantes es posible educar y trabajar en el desarrollo de actitudes no prejuiciosas a través de sus planes de estudios. Actualmente ya se han incorporado a los distintos programas universitarios españoles asignaturas y programas específicos para tratar estos fenómenos, como la asignatura de Transculturalidad, Género y Salud, que pretende desarrollar competencias sobre la apreciación de la diversidad y la multiculturalidad, conocimiento de culturas y costumbres de otras culturas, pero, a la vista de los resultados, éstos deberían ser más notorios en contextos con alta presión migratoria. Por lo que no es suficiente tratar las migraciones en una única asignatura. Durante la formación de los estudiantes se les instruye para saber cuidar a diferentes tipos de pacientes en función de la edad y de las características particulares de cada persona y aprenden a realizar planes de cuidados individualizados, por lo que sería necesario insistir en las diferencias culturales de los pacientes migrantes de manera transversal en todas las asignaturas específicas de enfermería.

Igualmente los resultados nos han permitido establecer un grupo clave en el que se recomienda centrar los esfuerzos (estudiante hombre de enfermería, de procedencia europea, de último curso y que no ha tenido contacto con inmigrantes) cuyo único objetivo sería facilitar la labor de enfermería que desarrollarán en los próximos años.

Es necesario señalar algunas limitaciones de esta investigación, en primer lugar, los participantes se seleccionaron con 
un muestreo no probabilístico por conveniencia y sería aconsejable realizarlo por cuotas, para representar a la población que conforman los universitarios matriculados de la Universidad de Granada (España), lo que puede conllevar la posibilidad de la generalización de los resultados obtenidos. En relación con esto, sería interesante realizar la investigación a todos los estudiantes del Grado en Enfermería en los distintos Campus de la Universidad de Granada (España) es decir, al alumnado que estudia en contextos multiculturales por su situación geográfica (Ceuta y Melilla, ciudades españolas situadas en el Norte de África) y Granada (cuyo contexto está alejado de la presión migratoria que viven Ceuta y Melilla) para poder comparar si las diferentes realidades influyen en los prejuicios de los futuros profesionales de Enfermería.

\section{RESUMEN}

Objetivo: Conocer si los estudiantes de enfermería poseen prejuicios sutiles y manifiestos hacia los migrantes. Método: Se realizó un estudio Ex-Post-Facto de tipo descriptivo. Las variables de estudio fueran: sexo, procedencia cultural, edad, curso y tener contacto con migrantes. Se utilizó la Escala de Prejuicio Sutil y Manifiesto. Se realizaron análisis descriptivos e inferenciales. Resultados: Participaron 282 estudiantes. Existen diferencias significativas en la variable cultura, siendo los estudiantes de procedencia bereber, de religión musulmana, menos prejuiciosos que los de procedencia europea, de religión cristiana. Los alumnos de cuarto, son los que más prejuicios manifiestos poseen. Según la variable haber tenido contacto con migrantes, son los estudiantes que no han tenido contacto con este colectivo los que tienen mayores prejuicios manifiestos. Conclusión: Los estudiantes de enfermería poseen cierta carga de prejuicios sutiles más que de prejuicios manifiestos hacia la población migrante.

\section{DESCRIPTORES}

Estudiantes de Enfermería; Migrantes; Prejuicio; Actitud; Atención de Enfermería.

\section{RESUMO}

Objetivo: Conhecer se os estudantes de enfermagem possuem preconceitos sutis e manifestos em relação aos migrantes. Método: Foi realizado um estudo Ex-Post-Facto de tipo descritivo. As variáveis de estudo foram: sexo, procedência cultura, idade, curso e ter contato com migrantes. Foi utilizada a Escala de Preconceito Sutil e Manifesto. Foram realizadas análises descritivas e inferenciais. Resultados: Participaram 282 estudantes. Existem diferenças significativas na variável cultura, sendo os estudantes de procedência berbere, de religião muçulmana, menos preconceituosos que os de procedência europeia, de religião cristã. Os alunos do quarto ano são os que mais têm preconceitos manifestos. De acordo com a variável ter tido contato com migrantes, são os estudantes que não tiveram contato com esse coletivo os que têm maiores preconceitos manifestos. Conclusão: Os estudantes de enfermagem possuem certa carga de preconceitos sutis mais do que de preconceitos manifestos em relação à população migrante.

\section{DESCRITORES}

Estudante de Enfermagem; Migrantes; Preconceito; Atitude; Cuidados de Enfermagem.

\section{REFERENCIAS}

1. Checa Olmos JC, Arjona Garrido A. Immigrants as seen by the Spanish: between threat and competition (1997-2007). Rev Estud Soc [Internet]. 2013 [cited 2018 Apr 2];(47):118-32. Available from: http://www.scielo.org.co/scielo.php?script=sci_abstract\&pid=S0123885X2013000300010

2. Alemany I, Mesa MC. Las relaciones intergrupales y su relación con las actitudes, estereotipos y prejuicios. En: Alemany I, Jiménez S, Sánchez S, editores. Formación del profesorado para la diversidad cultural. Madrid: La Murallla; 2012. p. 63-87.

3. Alemany I, Robles MC, De la Flor-Alemany MA. Las actitudes, los prejuicios y los estereotipos. En: Gallardo Vigil MA, Alemany Arrebola I, editores. Las actitudes ante diversas realidades sociales: buenas prácticas para la educación inclusiva. Granada: Comares; 2019. p. 1-7.

4. Etxeberría F, Murua H, Arrieta E, Garmendia J, Etxeberría Murgiondo J. Secondary students prejudices towards immigration. Teor Educ. $2013 ; 25(2): 155-87$.

5. Pettigrew TF, Meertens RW. Subtle and blatant prejudice in Western Europe. Eur J Soc Psychol. 1995;1(25):57-75. DOI: 10.1002/ ejsp.2420250106

6. Civalero L, Alonso D, Brussino S. Evaluación del prejuicio hacia inmigrantes: adaptación argentina de la escala de prejuicio sutil y manifiesto. Cienc Psicol. 2019;13(1):119-33. DOI: 10.22235/cp.v13i1.1814

7. León del Barco B, Mira AR, Gómez Carroza T. Evaluación de las opiniones sobre la inmigración y la multiculturalidad en la escuela de alumnos de magisterio. Rev Electrón Investig Psicoeduc Psicogedag. 2007;5(2):259-82.

8. Pettigrew TF, Wagner $U$, Christ $\mathrm{O}$, Stellmacher J. Direct and indirect intergroup contact effects on prejudice: a normative interpretation. Int J Intercult Relat. 2007;31(4):411-25.

9. Pettigrew TF, Tropp LR. A meta-analytic test of intergroup contact theory. J Pers Soc Pyschol. 2006;90(5):751-783. DOI: 10.1037/00223514.90.5.751

10. Guardia L, Nacarí G. Discriminación racial dentro del ámbito universitario: un enfoque sobre la discriminación sutil y manifiesta. Rei Do Crea. 2013;2:111-6.

11. Segura-Robles A, Alemany-Arrebola I, Gallardo-Vigil MÁ. Prejudiced attitudes of university students toward immigrants in an irregular situation: an exploratory study. Electron J Res Educ Psychol. 2016;14(39):393-416. DOI: http://dx.doi.org/10.14204/ejrep.39.15069

12. Sánchez Ortuño M, Campillo Cascales MJ, Illán Franco S, Llor Zaragoza L, Ruiz Hernández JA. Actitudes de una muestra de estudiantes de la región de Murcia hacia los inmigrantes magrebíes. Enferm Glob [Internet]. 2005 [citado 2018 Oct 15];4(2):1-9. Disponible en: http:// www.redalyc.org/articulo.oa?id=365834730012 
13. Regina M. Ensuring a unified and cross-cultural nursing language system the challenge faced by nursing terminology development. Rev Esc Enferm USP. 2015;49(4):535-9. DOI: http://dx.doi.org/10.1590/S0080-623420150000400001

14. Mora Guillart L. Los principios éticos y bioéticos aplicados a la calidad de la atención en enfermería. Rev Cubana Oftalmol [Internet]. 2015 [citado 2019 Abr 22];28(2):228-33. Disponible en: http://scielo.sld.cu/scielo.php?script=sci_arttext\&pid=S0864$21762015000200009 \& \operatorname{lng}=\mathrm{es}$

15. Flood JL, Commendador KA. Undergraduate nursing students and cross-cultural care: a program evaluation. Nurse Educ Today. 2016;36:190-4. DOI: 10.1016/j.nedt.2015.10.003

16. Ungaretti J, Etchezahar E, Barreiro A. Validation of the subtle and blatant prejudice scale towards indigenous people in Argentina. Curr Psychol. 2018;1(1):1-7. DOI: 10.1007/s12144-018-9844-4

17. Falanga R, Caroli M, Sagone E. Subtle and blatant prejudice toward Africans in Italian adolescents. Procedia Soc Behav Sci. 2014;116:70812.

18. Sijtsma K. Reliability beyond theory and into practice. Psychometrika. 2008;74(1):169-73. DOI: 10.1007/s11336-008-9103-y

19. Afthanorhan A, Aimran A, Ahmad S. Permutation test, non-parametric, and confidence set approaches to multi group analysis for comparing 2 Groups Using Partial Least Square Structural Equation Modeling (Pls-Sem). Adv Res. 2015;4(5):315-28.

20. Sedgwick P. A Comparison of parametric and non-parametric statistical tests. BMJ. 2015; 350:h2053. DOI: https://doi.org/10.1136/bmj. h2053

21. Derrac J, García S, Molina D, Herrera F. A practical tutorial on the use of nonparametric statistical tests as a methodology for comparing evolutionary and swarm intelligence algorithms. Swarm Evol Comput. 2011;1(1):3-18.

22. Passini S. Subtle prejudice and conformism: the intergroup indifference. Int J Psychol Res. 2017;10(1):25-34.

23. Cuevas R, Contreras O, García-Calvo T, Pastor JC. Nature and profiles of racial prejudice: analyses and educational implications in Secondary Scholl students. Infanc Aprendiz. 2014;37:248-77. DOI: 10.1080/02103702.2014.918815

24. García MC, Navas MS, Cuadrado I, Molero F. Immigration and prejudice: attitudes from a simple of adolescents in Almeria (Spain). Acción Psicol. 2003;2:137-47.

25. Asma M, Lindsay R. Immigration attitudes amongst European Muslims: social identity, economic threat and familiar experiences. Ethn Racial Stud. 2018;42(7):1050-69. DOI: 10.1080/01419870.2018.1472387

26. Moreno-Preciado M. Nurse/immigrant patient relation: research perspectives. Index Enferm. 2005;14(50):25-9.

27. Keshet Y, Popper-Giveon A. Race-based experiences of ethnic minority health professionals: Arab physicians and nurses in Israeli public healthcare organizations. Ethn Health. 2017;23(4):442-59.

28. Sánchez-Ojeda MA, Alemany-Arrebola I, Gallardo-Vigil MA. The nursing department's view towards moroccan patients. Rev Esc Enferm USP. 2017;51:e03227. DOI: http://dx.doi.org/10.1590/S1980-220X2016022703227

29. Allport GW. The open system in personality theory. J Abnorm Soc Psychol. 1960;61(3):301-10. DOI: 10.1037/h0043619

30. Hamberger J, Hewstone M. Tests of a model in four West European nations. Br J Soc Psychol. 1997;36:173-190. DOI: https://doi. org/10.1111/j.2044-8309.1997.tb01126.x

31. Herrero Olaizola J, Rodríguez Díaz FJ, Musitu Ochoa G. Comparing intergroup contact effects on blatant and subtle prejudice in adolescents: a multivariate multilevel model. Psicothema. 2014;26(1):33-8.

32. Pettigrew TF. Intergroup contact theory. Annu Rev Psychol. 1998;49:65-8. DOI: https://doi.org/10.1146/annurev.psych.49.1.65

33. Dhont K, Roets A, van Hiel A. Opening closed minds: The combined effects of intergroup contact and need for closure on prejudice. Pers Soc Psychol Bull. 2011;37(4):514-28. 